\title{
The information loss for QCD matter in mini black holes at LHC
}

\author{
Mohammad Ebrahim Zomorrodian, Alireza Sepehri, and Aliakbar Moradi Marjaneh
}

\begin{abstract}
In this paper we find information loss in mini black holes at LHC by extending the Horowitz and Maldacena mechanism to gluons and calculate the information transformation from the collapsing matter to the state of outgoing Hawking radiation for gluons, quarks, and mesons. Next, we calculate the total cross-section for mesons produced from black holes at LHC. We conclude that the more the mass of the quark within the hadrons, the lower is the cross-section. Then, we consider the effect of quarks inside the black hole on the density matrix of individual quarks within the meson outside of a black hole. At the end, we show that information is not lost in black holes if we ignore interactions between particles inside the event horizon in comparison with the effect of a black hole on particles. However, we observe that the unstable condition of excited black holes causes information loss in them. After that, we anticipate quantum black hole production by using electron-positron annihilation modeling at $\mathrm{TeV}$ centre of mass energies and compare the corresponding cross-sections calculated, at this stage, for different black hole states. Finally, we calculate the dependence of gluon tree level amplitudes to their thermal distributions near mini black holes at LHC.
\end{abstract}

PACS No: 04.70Dy

\begin{abstract}
Résumé : Nous étudions ici la perte d'information dans les mini trous noirs au LHC en étendant le mécanisme de Horowitz et Maldacena aux gluons et calculons la transformation d'information de l'effondrement de la matière jusqu'à la radiation sortante de Hawking, pour les gluons, les quarks et les gluons. Nous calculons ensuite la section efficace totale pour les mésons produits par les trous noirs au LHC. Nous trouvons que plus la masse du quark dans le hadron est élevée, plus la section efficace est faible. Nous considérons ensuite l'effet du de la présence du quark dans le trou noir sur la matrice de densité des quarks individuels à l'intérieur du méson hors du trou noir. Enfin, nous montrons que l'information n'est pas perdue dans les trous noirs si nous ignorons les interactions entre les particules à l'intérieur de l'horizon, en comparant avec l'effet du trou noir sur les particules. Cependant, nous constatons que l'instabilité des trous noirs excités cause une perte d'information. Par la suite, nous prévoyons la production de trous noirs quantiques en utilisant un modèle d'annihilation électron-posiron à l'échelle des $\mathrm{TeV}$ dans le centre de masse et comparons les sections efficaces calculées à ce point-ci pour différents états finals du trou noir. Pour finir, nous calculons la dépendance des amplitudes de gluons au niveau des arbres sur les distributions thermiques près des mini trous noirs au LHC.
\end{abstract}

[Traduit par la Rédaction]

\section{Introduction}

Recently Horowitz and Maldacena have suggested a mechanism to reconcile the unitarity of black hole evaporation [1]. But they did not make use of curved space-time. In this proposal, there were three different Hilbert spaces that belong to degrees of freedom of matter, incoming, and outgoing radiation. The total state of a black hole is a direct product of matter state and the entangled states of the states inside and outside of the event horizon. When a black hole begins to evaporate, the matter state will be in a maximally entangled state with incoming Hawking radiation. To describe the unknown effects of quantum gravity, an additional unitary transformation $\mathrm{S}$ is introduced. The outside state of a black hole is easily obtained by projecting this entangled state on the total state of the black hole (HM mechanism) [1]. So we want to use this mechanism for black hole states at LHC.

Recently, a model for quantum black hole production and decay in proton-proton collisions has been constructed [2, 3]. As the black hole masses at the LHC are relatively small (3-7 TeV), and the temperatures of the black holes are very high $(1 \mathrm{TeV})$, the black holes can be a source for quark production via Hawking radiation. In fact, there can be an enormous amount of heavy (supersymmetry and Higgs) particle production from black holes [4, 5], much more than expected from normal PQCD processes [6].

Generically speaking, quantum black holes (QBHs) form representations of $\mathrm{SU}(3)_{\mathrm{C}}$ and carry a QED charge. The process of two partons $P_{i}, P_{j}$ forms a quantum black hole in

Received 19 March 2010. Accepted 7 September 2010. Published on the NRC Research Press Web site at cjp.nrc.ca on 13 November 2010.

M.E. Zomorrodian, A. Sepehri, ${ }^{1,2}$ and A. Moradi Marjaneh. Ferdowsi University of Mashhad, Department of Physics, Mashhad 91775-1436, Iran.

${ }^{1}$ Corresponding author (e-mail: alireza.sepehri3@gmail.com).

${ }^{2}$ Alternate e-mail address: al_se36@yahoo.com. 
the $c$ representation of $\mathrm{SU}(3)_{\mathrm{C}}$ and charge $q$ : $P_{i}+P_{j} \rightarrow Q B H_{c}^{q}$. The following different transitions are possible at a proton collider [2, 3]:

(a) $3 \otimes \overline{3}=8 \oplus 1$

(b) $3 \otimes 3=6 \oplus \overline{3}$

(c) $3 \otimes 8=3 \oplus \overline{6} \oplus 15$

(d) $8 \otimes 8=1_{S} \oplus 8_{S} \oplus 8_{A} \oplus 10_{S} \oplus 1 \overline{0}_{A} \oplus 27_{S}$

(e) $\overline{3} \otimes \overline{3}=\overline{6} \oplus 3$

(f) $\overline{3} \otimes 8=\overline{3} \oplus 6 \oplus 1 \overline{5}$

However, we will show that the process of electron-positron annihilation at $\mathrm{TeV}$ centre of mass energies forms a quantum black hole without any color or charge $e^{+} e^{-} \rightarrow Q B H_{0}^{0}$.

In Sects. 2 and 3, we extend the Horowitz and Maldacena mechanism to gluons and quarks and calculate the information transformation from the collapsing matter to the state of the outgoing Hawking radiation for gluons and quarks. The meson production cross-section is calculated in Sect. 4. In this section we also study the effect of quarks inside the black hole on the density matrix of individual quarks within the meson outside of the black hole. Next, in Sect. 5, we observe that information loss is complete in the case of excited black holes. We predict the quantum black hole production by using electron-positron annihilation modeling at $\mathrm{TeV}$ centre of mass energies in Sect. 6. Finally, we discuss the tree level amplitudes of gluons near mini black holes at LHC.

\section{Information loss for gluons produced of black holes at LHC}

Now we want to extend the Horowitz and Maldacena mechanism to particles with spin 1, such as gluons. Previously it has been shown that the Minskowski vacuum will evolve into a state, called the Unruh state, which can be formulated as [7]

$|0\rangle_{\omega, \boldsymbol{p}, s}^{M}=\sqrt{\frac{2 \sinh (4 \pi M \omega)}{\mathrm{e}^{(4 \pi M \omega)}}} \sum \mathrm{e}^{-4 n \pi M \omega}|n(\omega, \boldsymbol{p}, s)\rangle_{\text {in }} \otimes|n(\omega,-\boldsymbol{p}, s)\rangle_{\text {out }}$

where $|n(\omega, \boldsymbol{p}, s)\rangle_{\text {in }}$ and $|n(\omega,-\boldsymbol{p}, s)\rangle_{\text {out }}$ denote the state with $n$ particles inside and outside of the black hole, and $\omega, \boldsymbol{p}, s$ are the particle's energy, momentum, and spin inside and outside of black hole, respectively. By using of this expression we can discuss the final state of the black hole.

Before black hole evaporation, there is an entanglement between the inside and outside of the horizon, but matter itself is not entangled [7-10],

$$
\begin{aligned}
& \left.\left.|g\rangle_{\text {in } \otimes \text { out }}=\sqrt{\frac{2 \sinh (4 \pi M \omega)}{\mathrm{e}^{(4 \pi M \omega)}}} \sum \mathrm{e}^{-4 n \pi M \omega} \mid n(\omega, \boldsymbol{p}, s, \text { color })\right\rangle_{\text {in }} \otimes \mid n(\omega,-\boldsymbol{p}, s, \text { anticolor })\right\rangle_{\text {out }} \\
& |g\rangle_{M \otimes \text { in } \otimes \text { out }}=|g\rangle_{M} \otimes|g\rangle_{\text {in } \otimes \text { out }}
\end{aligned}
$$

The sum is over $n$ and all color octets, which can be written as a basis of colored and anticolored states. After evaporation, we can describe the state of the black hole as an entangled state of both matter and the inside of the black hole,

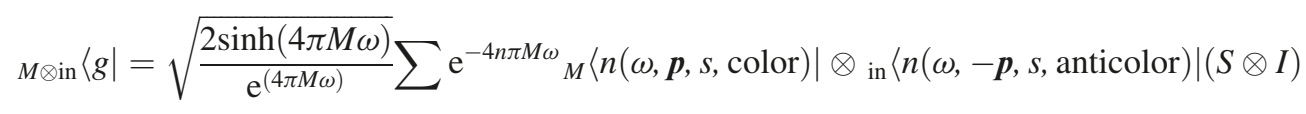

The outside state of the black hole is easily obtained by projecting (3) on the total state of the black hole in (2),

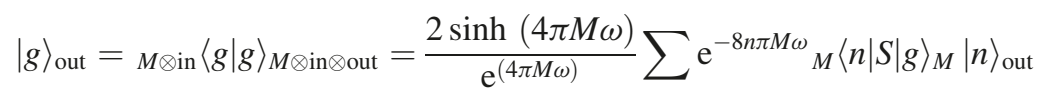

The information loss in a black hole has been a serious challenge to modern physics, because it contradicts the basic principles of quantum mechanics. This argument predicts that a process of black hole formation and evaporation is not unitary. Gottesman and Preskill explained how interactions between the collapsing body and infalling Hawking radiation inside the event horizon could cause a loss of information. They obtained the amount of this information loss [11].

Gottesman and Preskill introduce a unitary transformation $U$ to describe interactions between matter and the inside of the horizon $[11,12]$. In their fanciful language, the transformation $U$ can be interpreted as an interaction between the information's past and future. Various authors (Deutsch [12,13], for example) have pointed out that such interactions can cause a breakdown of unitarity,

$$
M \otimes \text { in }\langle g|=\sqrt{\frac{2 \sinh (4 \pi M \omega)}{\mathrm{e}^{(4 \pi M \omega)}}} \sum \mathrm{e}^{-4 n \pi M \omega}{ }_{M}\langle n(\omega, \boldsymbol{p}, s, \text { color })| \otimes{ }_{\text {in }}\langle n(\omega,-\boldsymbol{p}, s, \text { anticolor })|(S \otimes I) U
$$

Now we can calculate the information transformation $[14,15]$ from the collapsing matter to the state of the outgoing Hawking radiation for gluon fields as the following:

$$
\begin{aligned}
T_{g} & ={ }_{M \otimes \text { in }}\langle g \mid g\rangle_{\text {in } \otimes \text { out }}=\frac{2 \sinh (4 \pi M \omega)}{\mathrm{e}^{4 \pi M \omega}} \sum \mathrm{e}^{-8 \pi n M \omega}{ }_{M}\left\langle\left. n\right|_{\text {in }}\langle n|S \otimes U| n\rangle_{\text {in }} \mid n\right\rangle_{\text {out }} \\
& =\frac{2 \sinh (4 \pi M \omega)}{\mathrm{e}^{4 \pi M \omega}} \sum \mathrm{e}^{-8 \pi n M \omega}{ }_{M}\left\langle\left. n\right|_{\text {in }}\langle n|S \otimes U| n\rangle_{\text {in }} \mid n\right\rangle_{\text {out }}
\end{aligned}
$$




$$
\begin{aligned}
& f=\left|T_{g}\right|^{2}=\frac{4 \sinh ^{2}(4 \pi M \omega)}{\mathrm{e}^{8 \pi M \omega}} \sum_{n, n^{\prime}} \mathrm{e}^{-8 \pi M \omega\left(n+n^{\prime}\right)}{ }_{M}\left\langle\left. n\right|_{\text {in }}\left\langle\left. n\right|_{\text {in }}\left\langle\left. n^{\prime}\right|_{\text {out }}\left\langle n^{\prime}\left|S S^{t} \otimes U U^{t}\right| n^{\prime}\right\rangle_{\text {in }} \mid n^{\prime}\right\rangle_{M} \mid n\right\rangle_{\text {in }} \mid n\right\rangle_{\text {out }} \\
& =\frac{4 \sinh ^{2}(4 \pi M \omega)}{\mathrm{e}^{8 \pi M \omega}} \sum_{n, n^{\prime}} \mathrm{e}^{-8 \pi M \omega\left(n+n^{\prime}\right)}{ }_{M}\left\langle n \mid n^{\prime}\right\rangle_{M \text { in }}\left\langle n \mid n^{\prime}\right\rangle_{\text {in in }}\left\langle n^{\prime} \mid n\right\rangle_{\text {in out }}\left\langle n^{\prime} \mid n\right\rangle_{\text {out }} \\
& =\frac{4 \sinh ^{2}(4 \pi M \omega)}{\mathrm{e}^{8 \pi M \omega}} \sum_{n} \mathrm{e}^{-16 \pi M n \omega}=\frac{\left(1-\mathrm{e}^{-8 \pi M \omega}\right)^{2}}{\left(1-\mathrm{e}^{-16 \pi M \omega}\right)}=\frac{1-\mathrm{e}^{-8 \pi M \omega}}{1+\mathrm{e}^{8 \pi M \omega}}
\end{aligned}
$$

If the information transformation from the collapsing matter to the state of the outgoing Hawking radiation is complete, the value of $f$ should be one [12]. Thus we observe that because of the deviation of $f$ from unity, some information is lost in black holes. Similar calculations for quark fields will be done in next section.

\section{Information loss for quarks produced in black holes at LHC}

For quarks we can repeat the calculations in refs. 8-10 for Dirac fields that include color. Before black hole evaporation, there is entanglement between the inside and outside of the horizon, but matter itself is not entangled,

$$
\begin{aligned}
& \left.\left.|q\rangle_{\text {in } \otimes \text { out }}=\cos \left(r_{\omega}\right) \sum_{l=0,1} \mathrm{e}^{-4 \pi M l \omega} \mid k(\omega, \uparrow, \text { color })\right\rangle_{\text {in }} \otimes \mid k(\omega, \downarrow, \text { anticolor })\right\rangle_{\text {out }} \\
& |q\rangle_{M \otimes \text { in } \otimes \text { out }}=|q\rangle_{M} \otimes|q\rangle_{\text {in } \otimes \text { out }} \\
& |q\rangle_{M}=\sum_{l=0}^{1} A_{l}|l\rangle
\end{aligned}
$$

where

$$
\tan \left(r_{\omega}\right)=\mathrm{e}^{-4 \pi M \omega}, \quad \cos \left(r_{\omega}\right)=\left(1+\mathrm{e}^{-8 \pi M \omega}\right)^{-1 / 2}, \quad \sum_{l=0}^{1}\left|A_{l}\right|^{2}=1
$$

After evaporation, we can describe the state of the black hole as an entangled state of both matter and the inside of the black hole,

$$
M \otimes \text { in }\langle q|=\cos \left(r_{\omega}\right) \sum_{l, \text { color }} \mathrm{e}^{-4 \pi m \omega l}{ }_{M}\langle l(\omega, \uparrow, \text { color })| \otimes{ }_{\text {in }}\langle l(\omega, \downarrow, \text { anticolor })|(S \otimes I)
$$

where $S$ is a unitary transformation that acts on Dirac matter.

The outside state of the black hole is easily obtained by projecting the (8) on the total state of the black hole in $(7)[9,10]$,

$$
\left.\left|q_{0}\right\rangle_{\text {out }}=\cos ^{2}\left(r_{\omega}\right) \sum_{\text {color }=1}^{3} \sum_{l=0}^{1} \tan ^{2 l}\left(r_{\omega}\right){ }_{M}\langle l|S| q\rangle_{M} \mid l(\omega, \uparrow, \text { color })\right\rangle_{\text {out }}
$$

Now consider a pair of quarks that are created near the event horizon, and one of them falls in to the black hole. The ground state of the pair of particles is,

$$
\left|q_{0}\right\rangle_{\text {in } \otimes \text { out }}=\left(\cos (r)+\mathrm{e}^{-i \varphi} \sin (r) c_{k, \text { color }}^{\text {in }} d_{-k, \text { anticolor }}^{\text {out }}\right)|0\rangle_{\text {in }}^{-}|0\rangle_{\text {out }}^{+}
$$

where $c_{k, \text { color }}^{\text {in }}, d_{-k \text {,anticolor }}^{\text {out }}$ are annihilation operators that act on both inside states and outside states. We speak only about one special color. Using creation operators on (10), we have $[9,10]$,

$\left|q_{1}\right\rangle_{\text {in } \otimes \text { out }}=\left|1_{k}\right\rangle_{\text {out }}^{+}\left|0_{-k}\right\rangle_{\text {in }}^{-}$

where $+(-)$ denote particles (antiparticles). The state of the black hole evolves as

$\left|q^{\prime}\right\rangle_{M \otimes \text { in }}=\cos \left(r_{\omega}\right)\left|0_{k}\right\rangle_{M t}^{+}\left|0_{-k}\right\rangle_{\text {in }}^{-}+\sin \left(r_{\omega}\right)\left|1_{k}\right\rangle_{M}^{+}\left|1_{-k}\right\rangle_{\text {in }}^{-}$

On the other hand, in general we can write

$M \otimes$ in $\langle q|={ }_{M \otimes \text { in }}\left\langle q^{\prime}\right| S \otimes I$

The state of the system is

$$
\left|q_{i}\right\rangle_{M \otimes \text { in } \otimes \text { out }}=|q\rangle_{M} \otimes\left|q_{i}\right\rangle_{\text {in } \otimes \text { out }} \quad i=0,1
$$


Suppose that the state of the matter before evaporation is $|q\rangle_{M}$. The state of the field outside the black hole after evaporation becomes,

$\left|q_{1}\right\rangle_{\text {out }}={ }_{M \otimes \text { in }}\left\langle q \mid q_{1}\right\rangle_{M \otimes \text { in } \otimes \text { out }}=\cos \left(r_{\omega}\right)_{M}\langle 0|S| q\rangle_{M}|1\rangle_{\text {out }}$

It is interesting to compare a new excited state with the initial ground state. It can be found by projecting the state (15) on the initial ground state,

$$
\begin{aligned}
{ }_{\text {out }}\left\langle q_{1} \mid q_{0}\right\rangle_{\text {out }} & =\cos ^{3}\left(r_{\omega}\right) \sum_{l=0}^{1} \tan ^{2 l}\left(r_{\omega}\right)_{M}\left\langle q\left|S^{t}\right| 0\right\rangle_{M}\langle l|S| q\rangle_{M} \text { out }\langle 1 \mid l\rangle_{\text {out }}={ }_{M}\left\langle q\left|\cos ^{3}\left(r_{\omega}\right) \tan ^{2}\left(r_{\omega}\right) S^{t}\right| 0\right\rangle_{M}\langle 1|S| q\rangle_{M} \\
& ={ }_{M}\left\langle q\left|z^{2}\right| q\right\rangle_{M} \\
& =\sum_{l} \sum_{l}\left\langle l\left|A_{l} A_{l}^{*} \cos ^{3}\left(r_{\omega}\right) \tan ^{2}\left(r_{\omega}\right) S^{t}\right| 0\right\rangle_{M}\langle 1|S| l\rangle_{M} \\
& =\sum_{l}{ }_{M}\left\langle l\left|\cos ^{3}\left(r_{\omega}\right) \tan ^{2}\left(r_{\omega}\right) S^{t}\right| 0\right\rangle_{M}\left\langle\left. 1\left|\sum S\right| A_{l}\right|^{2} \mid l\right\rangle_{M} \\
& =\operatorname{Tr}\left[\cos ^{3}\left(r_{\omega}\right) \tan ^{2}\left(r_{\omega}\right) S^{t}|0\rangle_{M M}\langle 1| S\right]=\cos ^{3}\left(r_{\omega}\right) \tan ^{2}\left(r_{\omega}\right) \operatorname{Tr}\left[S^{t} S|0\rangle_{M}\langle 1|\right] \\
& =\cos ^{3}\left(r_{\omega}\right) \tan ^{2}\left(r_{\omega}\right) \operatorname{Tr}\left[\rho_{M}\right]=\cos ^{3}\left(r_{\omega}\right) \tan ^{2}\left(r_{\omega}\right) \operatorname{Tr}\left[M\langle 0 \mid 1\rangle_{M}\right]=0
\end{aligned}
$$

where $z^{2}=\cos ^{3}\left(r_{\omega}\right) \tan ^{2}\left(r_{\omega}\right) S^{t} \rho_{M} S$ and $\rho_{M}=|0\rangle_{M M}\langle 1|$. Also we have used $\operatorname{Tr}[\mathrm{AB}]=\operatorname{Tr}[\mathrm{BA}]$.

From elementary quantum theory, the term ${ }_{M}\left\langle q\left|z^{2}\right| q\right\rangle_{M}$ in (16) is nothing but the expectation value of $z^{2}$ averaged over the initial matter state, which can also be obtained by the trace operation. A similar calculation has been done for the scalar field [8]. We observe that the final excited state out of the black hole for Dirac fields is orthogonal to the initial state before the process. This means that the final state of the fields outside the black hole is strongly affected by the evaporation process. In a manner similar to that used in (6) for quark fields we derive,

$$
\begin{gathered}
T_{q}={ }_{M \otimes \text { in }}\langle q \mid q\rangle_{\text {in } \otimes \text { out }}=\cos ^{2}\left(r_{\omega}\right) \sum \mathrm{e}^{-8 \pi n M \omega}{ }_{M}\left\langle\left. l\right|_{\text {in }}\langle l|S \otimes U| l\rangle_{\text {in }} \mid l\right\rangle_{\text {out }} \\
=\cos ^{2}\left(r_{\omega}\right) \sum \mathrm{e}^{-8 \pi n M \omega}{ }_{M}\left\langle\left. l\right|_{\text {in }}\langle l|S \otimes U| l\rangle_{\text {in }} \mid l\right\rangle_{\text {out }} \\
f=\left|T_{q}\right|^{2}=\cos ^{4}\left(r_{\omega}\right) \sum_{l, l^{\prime}} \mathrm{e}^{-8 \pi M \omega\left(l+l^{\prime}\right)}{ }_{M}\left\langle\left. l\right|_{\text {in }}\left\langle\left. l\right|_{\text {in }}\left\langle\left. l^{\prime}\right|_{\text {out }}\left\langle l^{\prime}\left|S S^{t} \otimes U U^{t}\right| l^{\prime}\right\rangle_{\text {in }} \mid l^{\prime}\right\rangle_{M} \mid l\right\rangle_{\text {in }} \mid l\right\rangle_{\text {out }} \\
=\cos ^{4}\left(r_{\omega}\right) \sum_{l, l^{\prime}} \mathrm{e}^{-8 \pi M \omega\left(l+l^{\prime}\right)}{ }_{M}\left\langle l \mid l^{\prime}\right\rangle_{M \text { in }}\left\langle l \mid l^{\prime}\right\rangle_{\text {in in }}\left\langle l^{\prime} \mid l\right\rangle_{\text {in out }}\left\langle l^{\prime} \mid l\right\rangle_{\text {out }} \\
=\cos ^{4}\left(r_{\omega}\right) \sum_{l} \mathrm{e}^{-16 \pi M l \omega}=\frac{1+\mathrm{e}^{-16 \pi M \omega}}{\left(1+\mathrm{e}^{-8 \pi M \omega}\right)^{2}}
\end{gathered}
$$

Thus we observe that due to the deviation of $f$ from unity, some of information is lost in black holes for gluons and quarks. In fact, the observer outside of black hole does not have any access to the inside of the black hole and some of information is lost.

\section{Information loss for mesons produced in black holes at LHC}

In this section we consider the behavior of mesons near the event horizon of mini black holes at LHC. For the outside final state of black holes for mesons as scalar fields we have [8-10],

$\mid$ meson $\rangle_{\text {out }}=\frac{1}{\cosh ^{2}\left(r_{\omega}\right)} \sum \mathrm{e}^{-8 \pi M \omega n}{ }_{M}\langle n|S| \text { meson }\rangle_{M}|n\rangle_{\text {out }}$
$\tanh \left(r_{\omega}\right)=\mathrm{e}^{-4 \pi M \omega}, \quad \cosh \left(r_{\omega}\right)=\left(1-\mathrm{e}^{-8 \pi M \omega}\right)^{-(1 / 2)}$

where $\mid$ meson $\rangle_{\text {out }}$ is the initial quantum state of matter, $S$ is a unitary transformation, and $\omega$ is related to the energy of the meson.

Here we only consider the color part of the wave functions $\mid$ meson $\rangle$ and $\left\langle\right.$ meson| coming from the representation $3 \otimes 3^{*}$ for the color singlet wave functions of the mesons [16],

$\mid$ meson $\rangle=\frac{1}{\sqrt{3}}\left(\left|1_{A} 1_{A}^{*}\right\rangle_{M}+\left|1_{B} 1_{B}^{*}\right\rangle+\left|1_{C} 1_{C}^{*}\right\rangle\right)$

$$
|1\rangle_{M}=\left|1_{k}\right\rangle_{\text {out }}^{+}\left|0_{-k}\right\rangle_{\text {in }}^{-}
$$

Previously, it has been shown that the Minskowski state will evolve into a state, called the Unruh state, which can be formulated as [9, 10], 
where $|0\rangle_{\text {in }},|1\rangle_{\text {out }}$ denote the states, inside and outside of the black hole and $|1\rangle_{M}$ denotes the Minskowski state. Using (20) and (19) we can easily derive,

$\mid$ meson $\rangle_{M, S}=\frac{1}{\sqrt{3}}\left(\left|1_{A} 1_{A}^{*}\right\rangle_{\text {out }}\left|0_{A} 0_{A}^{*}\right\rangle_{\text {in }}+\left|1_{B} 1_{B}^{*}\right\rangle_{\text {out }}\left|0_{B} 0_{B}^{*}\right\rangle_{\text {in }}+\left|1_{C} 1_{C}^{*}\right\rangle_{\text {out }}\left|0_{C} 0_{C}^{*}\right\rangle_{\text {in }}\right)$

and keeping the left to right order of the quark and antiquark for its conjugate wave function,

${ }_{M, S}\langle$ meson $|=\frac{1}{\sqrt{3}}\left({ }_{\text {out }}\left\langle 1_{A} 1_{A}^{*}\right|\right.$ in $\left\langle 0_{A} 0_{A}^{*}\right|+{ }_{\text {out }}\left\langle 1_{B} 1_{B}^{*}\right|$ in $\left\langle 0_{B} 0_{B}^{*}\right|+$ out $_{C}\left\langle\left. 1_{C} 1_{C}^{*}\right|_{\text {in }}\left\langle 0_{C} 0_{C}^{*}\right|\right)$

We can now write down the density matrices $\rho$ for the hadrons using the direct product of $\mid$ meson $\rangle$ and $\langle$ meson $|$,

$\rho_{M, S}=|\operatorname{meson}\rangle_{M, S} M, S\langle$ meson $|$

For quarks, we look at the one quark reduced density matrices, which give the statistical state of the individual quark within the hadron. To get the reduced density matrices for the mesons, we project out all the antiquark states $\left|i^{*}\right\rangle$ and $\left\langle j^{*}\right|$ by using the orthonormality and the completeness properties,

$\rho_{q}=\frac{1}{3}\left(\left|1_{\text {out }} 0_{\text {in }}\right\rangle_{A A}\left\langle 1_{\text {out }} 0_{\text {in }}|+| 1_{\text {out }} 0_{\text {in }}\right\rangle_{B B}\left\langle 1_{\text {out }} 0_{\text {in }}|+| 1_{\text {out }} 0_{\text {in }}\right\rangle_{C} C\left\langle 1_{\text {out }} 0_{\text {in }}\right|\right)$

This is the reduced density matrix for the quarks in the color singlet state. To calculate density matrices for the outside state, we project out all the inside states $|0\rangle_{\text {in }}$ and out $\langle 0|$ by using the orthonormality properties,

$\rho_{q}^{\text {out }}=\frac{1}{3}\left(\left|1_{\text {out }}\right\rangle_{A A}\left\langle 1_{\text {out }}|+| 1_{\text {out }}\right\rangle_{B B}\left\langle 1_{\text {out }}|+| 1_{\text {out }}\right\rangle_{C C}\left\langle 1_{\text {out }}\right|\right)$

This density matrix is similar to that in ref. 16. Thus, we conclude that near black holes the color density inside the mesons does not change, because there is no effect by the quarks inside the black hole on the density matrix of individual quarks within the meson.

The entropy $S$ of the quantum states calculated using this density matrix becomes $S=\ln 3$. This value of $S$ is equal to the entropy of the quark density of mesons in flat space. In fact, near a black hole, there is no decrease in entanglement between quarks inside meson. For this reason, the color structure of mesons does not change in curved space-time. The black holes at LHC can emit these mesons; this leads to the fast decay of the black holes themselves.

Now we calculate the total cross-section for mesons as scalar fields produced from a black hole,

$\sigma_{\text {meson }}^{1}=\frac{1}{3}\left(N_{\text {quark }}^{A} N_{\text {antiquark }}^{A}+N_{\text {quark }}^{B} N_{\text {antiquark }}^{B}+N_{\text {quark }}^{C} N_{\text {antiquark }}^{C}\right) \sigma_{\mathrm{BH}}$

in which

$N_{\text {quark }}=\int_{0}^{t_{f}} \mathrm{~d} t \int_{0}^{M_{\mathrm{BH}}} \mathrm{d} p \frac{C_{S} \sigma_{S}}{8 \pi^{2}} \frac{P^{2}}{\left[\exp \left(\frac{\sqrt{p^{2}+M_{\text {quark }}^{2}}}{T_{\mathrm{BH}}}\right)+1\right]}$

$N_{\text {antiquark }}=\int_{0}^{t_{f}} \mathrm{~d} t \int_{0}^{M_{\mathrm{BH}}} \mathrm{d} p \frac{C_{S} \sigma_{S}}{8 \pi^{2}} \frac{P^{2}}{\left[\exp \left(\frac{\sqrt{p^{2}+M_{\text {antiquark }}^{2}}}{T_{\mathrm{BH}}}\right)+1\right]}$

$t_{f}=\frac{C}{M_{\mathrm{P}}}\left(\frac{M_{\mathrm{BH}}}{M_{\mathrm{P}}}\right)^{\frac{d+3}{d+1}}$

where $d$ is the number of extra dimensions, $\sigma_{s}$ is the grey-body factor $[6,17]$, and $C$ depends on the extra dimensions and also on the polarization degrees of freedom, etc. However, the complete determination of $t_{f}$ depends on the energy density present outside the black hole, which is computed in [17] where the absorption of the quark-gluon plasma [17] by a TeV scale black hole at the LHC is considered. The black hole production cross-section in a binary partonic collision is given by [18],

$\sigma^{a b \rightarrow \mathrm{BH}}=\frac{1}{M_{\mathrm{P}}^{2}}\left\{\frac{M_{\mathrm{BH}}}{M_{\mathrm{P}}}\left[\frac{8 \Gamma((d+3) / 2)}{d+2}\right]\right\}^{2 /(d+1)}$ 
By using the quark masses we can compare different production cross-sections with each other,

$\sigma_{u \bar{u}} \geq \sigma_{d \bar{d}}>\sigma_{s \bar{s}}>\sigma_{c \bar{c}}>\sigma_{b \bar{b}}>\sigma_{t \bar{t}}$

Furthermore, some mesons, such as pions, may form in the matter of a black hole and then exit from the black hole. Their cross-sections are calculated as follows:

$\sigma_{\text {meson }}^{w}=N_{\text {meson }} \sigma_{\mathrm{BH}}$

in which

$N_{\text {meson }}=\int_{0}^{t_{f}} \mathrm{~d} t \int_{0}^{M_{\mathrm{BH}}} \mathrm{d} p \frac{C_{S} \sigma_{S}}{8 \pi^{2}} \frac{P^{2}}{\left[\exp \left(\frac{\sqrt{p^{2}+M_{\text {meson }}^{2}}}{T_{\mathrm{BH}}}\right)+1\right]}$

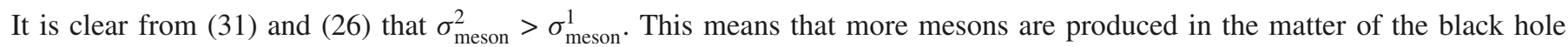
and then come out of the black hole. However, we should regard information loss in our calculation.

We can calculate the resulting information transformation from the collapsing matter to the state of the outgoing Hawking radiation for mesons,

$$
\begin{aligned}
& \mid \text { meson }\rangle_{\text {in } \otimes \text { out }}=\frac{1}{\cosh \left(r_{\omega}\right)} \sum \mathrm{e}^{-4 \pi M \omega n}|n\rangle_{\text {in }} \otimes|n\rangle_{\text {out }} \\
& M \otimes \text { in }\langle\text { meson }|=\frac{1}{\cosh \left(r_{\omega}\right)} \sum \mathrm{e}^{-4 \pi M \omega n}{ }_{M}\langle n| \otimes{ }_{\text {in }}\langle n|(S \otimes I) U \\
& \left.T_{\text {meson }}={ }_{M \otimes \text { in }}\langle\text { meson }| \text { meson }\right\rangle_{\text {in } \otimes \text { out }}=\frac{1}{\cosh ^{2}\left(r_{\omega}\right)} \sum \mathrm{e}^{-8 \pi n M \omega}{ }_{M}\left\langle\left. n\right|_{\text {in }}\langle n|S \otimes U| n\rangle_{\text {in }} \mid n\right\rangle_{\text {out }} \\
& =\frac{1}{\cosh ^{2}\left(r_{\omega}\right)} \sum \mathrm{e}^{-8 \pi n M \omega}{ }_{M}\left\langle\left. n\right|_{\text {in }}\langle n|S \otimes U| n\rangle_{\text {in }} \mid n\right\rangle_{\text {out }} \\
& f=\left|T_{\text {meson }}\right|^{2}=\frac{1}{\cosh ^{4}\left(r_{\omega}\right)} \sum_{n, n^{\prime}} \mathrm{e}^{-8 \pi M \omega(n+n \prime)}{ }_{M}\left\langle\left. n\right|_{\text {in }}\left\langle\left. n\right|_{\text {in }}\left\langle\left. n^{\prime}\right|_{\text {out }}\left\langle n^{\prime}\left|S S^{t} \otimes U U^{t}\right| n^{\prime}\right\rangle_{\text {in }} \mid n^{\prime}\right\rangle_{M} \mid n\right\rangle_{\text {in }} \mid n\right\rangle_{\text {out }} \\
& =\frac{1}{\cosh ^{4}\left(r_{\omega}\right)} \sum_{n, n^{\prime}} \mathrm{e}^{-8 \pi M \omega\left(n+n^{\prime}\right)}{ }_{M}\left\langle n \mid n^{\prime}\right\rangle_{M \text { in }}\left\langle n \mid n^{\prime}\right\rangle_{\text {in in }}\left\langle n^{\prime} \mid n\right\rangle_{\text {in out }}\left\langle n^{\prime} \mid n\right\rangle_{\text {out }} \\
& =\frac{1}{\cosh ^{4}\left(r_{\omega}\right)} \sum_{n} \mathrm{e}^{-16 \pi M \omega n}=\frac{\left(1-\mathrm{e}^{-8 \pi M \omega}\right)^{2}}{\left(1-\mathrm{e}^{-16 \pi M \omega}\right)}=\frac{1-\mathrm{e}^{-8 \pi M \omega}}{1+\mathrm{e}^{8 \pi M \omega}}
\end{aligned}
$$

We conclude that with increasing the mass of mesons the information loss increase. Thus most of mesons produced from these black holes are pions.

It seems that information is not lost in black holes if we assume that the black hole mass is very large,

$f=f_{\text {meson }} \times f_{\text {quark }} \times f_{\text {gluon }}=\left|\left(\frac{1-\mathrm{e}^{-8 \pi M \omega}}{1+\mathrm{e}^{-8 \pi M \omega}}\right)^{2} \frac{1+\mathrm{e}^{-16 \pi M \omega}}{\left(1-\mathrm{e}^{-8 \pi M \omega}\right)^{2}}\right|, \quad \lim _{M \rightarrow \infty} f=1$

This result is very important for considering black holes at LHC. This means that all of information inside the black hole can be transferred to outside of the black hole. It seems that interactions between particles inside the event horizon can be ignored when comparing the effect of a black hole on particles.

\section{Information loss in excited black holes}

In this section we suppose that a pair of particles are created near the event horizon and that one of them falls into the black hole. We want to calculate information loss for this black holes.

In excited black holes for gluons we have [7, 8],

$\left|g^{\prime}\right\rangle_{\text {in } \otimes \text { out }}=\left(1-\mathrm{e}^{-8 \pi M \omega}\right) \sum \mathrm{e}^{-4 n \pi M \omega} \sqrt{n+1} \mid n+1(\omega, \boldsymbol{p}, s$, color $\left.)\right\rangle_{\text {in }} \otimes \mid n(\omega,-\boldsymbol{p}, s$, anticolor $\left.)\right\rangle_{\text {out }}$

Now we can calculate information loss for this state, 


$$
\begin{aligned}
T_{g} & ={ }_{M \otimes \text { in }}\left\langle g \mid g^{\prime}\right\rangle_{\text {in } \otimes \text { out }}=\left(1-\mathrm{e}^{-8 \pi M \omega}\right)^{3 / 2} \sum \mathrm{e}^{-8 \pi n M \omega} \sqrt{n+1}_{M}\left\langle\left. n\right|_{\text {in }}\langle n|S \otimes U| n\rangle_{\text {out }} \mid n+1\right\rangle_{\text {in }} \\
f & =\left|T_{g}\right|^{2} \\
& =\left(1-\mathrm{e}^{-8 \pi M \omega}\right)^{3} \sum \mathrm{e}^{-8 \pi M \omega\left(n+n^{\prime}\right)} \sqrt{(n+1)\left(n^{\prime}+1\right)}{ }_{M}\left\langle\left. n^{\prime}\right|_{\text {in }}\left\langle\left. n^{\prime}\right|_{\text {out }}\left\langle\left. n\right|_{\text {in }}\left\langle n+1\left|S S^{t} \otimes U U^{t}\right| n^{\prime}+1\right\rangle_{\text {in }} \mid n^{\prime}\right\rangle_{\text {out }} \mid n\right\rangle_{\text {in }} \mid n\right\rangle_{M} \\
& =\left(1-\mathrm{e}^{-8 \pi M \omega}\right)^{3} \sum \mathrm{e}^{-8 \pi M \omega\left(n+n^{\prime}\right)} \sqrt{(n+1)\left(n^{\prime}+1\right)}\left\langle n^{\prime}|I \otimes I| n\right\rangle_{M} \text { in }\langle n+1 \mid n\rangle_{\text {in in }}\left\langle n^{\prime} \mid n^{\prime}+1\right\rangle_{\text {in out }}\left\langle n^{\prime} \mid n\right\rangle_{\text {out }} \\
& =0
\end{aligned}
$$

The result above shows that information is lost completely in excited black holes.

A similar calculation for quark fields and mesons can be done. For quark fields we derive [9, 10],

$\left|q^{\prime}\right\rangle_{\text {in } \otimes \text { out }}=\left|1_{k}\right\rangle_{\text {out }}^{+}\left|0_{-k}\right\rangle_{\text {in }}^{-}$

The amount of information loss for quarks is

$$
\begin{aligned}
& T_{q^{\prime}}={ }_{M \otimes \text { in }}\left\langle q \mid q^{\prime}\right\rangle_{\text {in } \otimes \text { out }}=\cos \left(r_{\omega}\right) \sum \mathrm{e}^{-4 \pi l M \omega}{ }_{M}\left\langle\left. l\right|_{\text {in }}\langle l|S \otimes U| 0\rangle_{\text {in }} \mid 1\right\rangle_{\text {out }} \\
& f=\left|T_{q}\right|^{2}=\cos ^{2}\left(r_{\omega}\right) \sum \mathrm{e}^{-4 \pi M \omega\left(l+l^{\prime}\right)}{ }_{M}\left\langle\left. l\right|_{\text {in }}\left\langle\left. l\right|_{\text {in }}\left\langle l^{\prime}-\left.1\right|_{\text {out }}\left\langle l^{\prime}\left|S S^{t} \otimes U U^{t}\right| l^{\prime}\right\rangle_{\text {in }} \mid l^{\prime}\right\rangle_{M} \mid l-1\right\rangle_{\text {in }} \mid l\right\rangle_{\text {out }} \\
&=\cos ^{2}\left(r_{\omega}\right) \sum \mathrm{e}^{-4 \pi M \omega\left(l+l^{\prime}\right)}{ }_{M}\left\langle l|I \otimes I| l^{\prime}\right\rangle_{M \text { in }}\left\langle l^{\prime}-1 \mid l^{\prime}\right\rangle_{\text {in in }}\langle l \mid l-1\rangle_{\text {in out }}\left\langle l^{\prime} \mid l\right\rangle_{\text {out }} \\
&=0
\end{aligned}
$$

Finally for mesons we write [8],

$\mid$ meson $\left.^{\prime}\right\rangle_{\text {in } \otimes \text { out }}=\frac{1}{\cosh ^{2}\left(r_{\omega}\right)} \sum \mathrm{e}^{-4 n \pi M \omega} \sqrt{n+1} \mid n+1(\omega, \boldsymbol{p}, s$, color $\left.)\right\rangle_{\text {in }} \otimes \mid n(\omega,-\boldsymbol{p}, s$, anticolor $\left.)\right\rangle_{\text {out }}$

Our calculations show,

$$
\begin{aligned}
& \left.T_{\text {meson }^{\prime}}={ }_{M \otimes \text { in }}\langle\text { meson }| \text { meson }^{\prime}\right\rangle_{\text {in } \otimes \text { out }}=\frac{1}{\cosh ^{3}\left(r_{\omega}\right)} \sum \mathrm{e}^{-4 \pi n M \omega} \sqrt{n+1}_{M}\left\langle\left. n\right|_{\text {in }}\langle l|S \otimes U| n+1\rangle_{\text {in }} \mid n\right\rangle_{\text {out }} \\
& f=\left|T_{\text {meson }}\right|^{2} \\
& =\frac{1}{\cosh ^{6}\left(r_{\omega}\right)} \sum \mathrm{e}^{-8 \pi M \omega\left(n+n^{\prime}\right)} \sqrt{(n+1)\left(n^{\prime}+1\right)}{ }_{M}\left\langle\left. n\right|_{\text {in }}\left\langle\left. n\right|_{\text {in }}\left\langle n^{\prime}+\left.1\right|_{\text {out }}\left\langle n^{\prime}\left|S S^{t} \otimes U U^{t}\right| n^{\prime}\right\rangle_{\text {in }} \mid n^{\prime}\right\rangle_{M} \mid n+1\right\rangle_{\text {in }} \mid n\right\rangle_{\text {out }} \\
& = \\
& =\frac{1}{\cosh ^{6}\left(r_{\omega}\right)} \sum \mathrm{e}^{-8 \pi M \omega\left(n+n^{\prime}\right)} \sqrt{(n+1)\left(n^{\prime}+1\right)}\left\langle n|I \otimes I| n^{\prime}\right\rangle_{M \text { in }}\langle n \mid n+1\rangle_{\text {in in }}\left\langle n^{\prime}+1 \mid n^{\prime}\right\rangle_{\text {in out }}\left\langle n^{\prime} \mid n\right\rangle_{\text {out }} \\
& =0
\end{aligned}
$$

We observe that information loss is complete in the case of excited black holes. The reason is the unstable condition of excited black holes. In these conditions we cannot use the Horowitz and Maldacena mechansim until black hole states become stable.

\section{Black hole production for electron-positron annihilation at TeV centre of mass energies}

We assume the process of electron-positron annihilation at $\mathrm{TeV}$ centre of mass energy that forma a quantum black hole without any color or charge. The possible quantum black hole states of matter of this interaction are $(0, q \bar{q})$. By applying the Horowitz and Maldacena mechanism we can calculate the outside state of the black hole,

$|q, \bar{q}\rangle_{\text {out }}=\left(\cos ^{2}\left(r_{\omega}\right) \sum_{l=0}^{1} \tan ^{2 l}\left(r_{\omega}\right)_{M}\langle l|s| q\rangle_{M}|l\rangle_{\text {out }}\right) \otimes\left(\cos ^{2}\left(r_{\omega^{\prime}}\right) \sum_{j=0}^{1} \tan ^{2 j}\left(r_{\omega^{\prime}}\right)_{M}\langle j|s| \bar{q}\rangle_{M}|j\rangle_{\text {out }}\right)$

To obtain the total cross-section from all black holes produced in electron-positron collisions, we need to multiply the black hole production cross-section with the number of quarks and gluons produced from a single black hole, 
$\sigma_{q, \bar{q}}=N_{q} N \bar{q} \sigma_{\mathrm{BH}}$

in which

$N_{q}=\int_{0}^{t_{f}} \mathrm{~d} t \int_{0}^{M_{\mathrm{BH}}} \mathrm{d} p \frac{C_{S} \sigma_{S}}{8 \pi^{2}} \frac{P^{2}}{\left(\exp \left[\frac{\sqrt{p^{2}+M_{q}^{2}}}{T_{\mathrm{BH}}}\right]+1\right)}$

and $\sigma_{\mathrm{BH}}$ was calculated in (29). Using (45), we can compare three jet cross-sections for different quark production,

$\sigma_{u, \bar{u}} \geq \sigma_{d, \bar{d}}>\sigma_{s, \bar{s}}>\sigma_{c, \bar{c}}>\sigma_{b, \bar{b}}>\sigma_{t, \bar{t}}$

In electron-positron annihilation, there are fewer quantum black hole states compared with proton-proton annihilation, because this interaction is neutral.

\section{Gauge theory color structure at LHC}

In this section, we consider the three amplitudes for gluon self-interactions at LHC. In proton-proton and electron-positron collisions, some gluons are produced produced because of transverse momentum between quarks and antiquarks. However in curved space-time, the near event horizon of mini black holes at LHC many gluons are produced becaause of the Unruh effect and interact with each other. In fact, the Minkowski vacuum for gluons will evolve into a state, called the Unruh state, which can be formulated as [7],

$|0\rangle_{M}=\sqrt{\frac{2 \sinh (4 \pi M \omega)}{\mathrm{e}^{(4 \pi M \omega)}}} \sum \mathrm{e}^{-4 \pi n M \omega} \mid n$, color $\rangle_{\text {in }} \otimes \mid n$, anticolor $\rangle_{\text {out }}$

In this equation $|n\rangle_{\text {in }}|n\rangle_{\text {out }}$ denote the states with $n$ gluons inside and outside of the black hole, $\omega$ is the gluon energy, and M is the mass of the black hole. Equation (48) shows that many gluons are produced near the event horizon of mini black holes at LHC. Of course the thermal distribution for gluon production is related to black hole mass and the energy of the gluons. The thermal distribution for these groups of gluons is

$$
\begin{aligned}
N_{\omega, \text { color }}^{\text {gluon }} & ={ }_{M}\left\langle 0\left|a_{\text {out, color }}^{t} a_{\text {out }, \text { color }}\right| 0\right\rangle_{M}={ }_{\text {out }}\left\langle\left. n\right|_{\text {in }}\left\langle n\left|\frac{2 \sinh (4 \pi M \omega)}{\mathrm{e}^{4 \pi M \omega}} a_{\text {out, color }}^{t} a_{\text {out }, \text { color }} \sum_{n=0}^{\infty} \mathrm{e}^{-8 \pi M n \omega}\right| n\right\rangle_{\text {in }} \mid n\right\rangle_{\text {out }} \\
& ={ }_{\text {out }}\left\langle n-\left.1\right|_{\text {in }}\left\langle n\left|\frac{2 \sinh (4 \pi M \omega)}{\mathrm{e}^{4 \pi M \omega}} \sum_{n=0}^{\infty} \mathrm{e}^{-8 \pi M n \omega} n\right| n\right\rangle_{\text {in }} \mid n-1\right\rangle_{\text {out }}={ }_{\text {out }}\left\langle n\left|\frac{2 \sinh (4 \pi M \omega)}{\mathrm{e}^{4 \pi M \omega}} \sum_{n=1}^{\infty} \mathrm{e}^{-8 \pi M n \omega}(n+1)\right| n\right\rangle_{\text {out }} \\
& =\frac{2 \sinh (4 \pi M \omega)}{\mathrm{e}^{4 \pi M \omega}} \sum_{n=1}^{\omega} \mathrm{e}^{-8 \pi M n \omega}(n+1)=\frac{2 \sinh (4 \pi M \omega)}{\mathrm{e}^{4 \pi M \omega}} \frac{1}{\left(1-\mathrm{e}^{-16 \pi M \omega}\right)^{2}}=\frac{1-\mathrm{e}^{-8 \pi M \omega}}{\left(1-\mathrm{e}^{-16 M \pi \omega}\right)^{2}}
\end{aligned}
$$

where $a_{\text {out,color }}^{t}, a_{\text {out,color }}$ are creation and annihilation operators that act on the black hole outside states of gluons. $N_{\omega, \text { color }}^{\text {gluon }}$ is the thermal distribution for gluons with energy $\omega$ and special color .

We now calculate tree amplitude for these two groups of gluons. At tree level, with gluons produced because of the transverse momentum between quarks and antiquarks, the full tree amplitude in the adjoint representation of $S U\left(N_{C}\right)\left(N_{c}=3\right.$ for QCD) can be decomposed as [19],

$$
\begin{array}{ll}
H_{n}^{\text {tree }}(1,2,3, \ldots ., n)=\alpha_{s}^{n-1} \sum_{p(2,3, \ldots, n)} \operatorname{Tr}\left[T^{a_{1}} T^{a_{2}} T^{a_{3}} \ldots . . T^{a_{n}}\right] A_{n}^{\text {tree }}(1,2,3, \ldots, n) & \\
A_{n}^{\text {tree }}(1,2,3, \ldots . n)=\sum_{j} \frac{n_{j}}{\left(\prod_{m} p_{m}^{2}\right) j} \quad\left[T^{a}, T^{b}\right]=\text { if }{ }^{a b c} T^{c}
\end{array}
$$

The sum in (50) is over all noncyclic permutations of legs, which is equivalent to all permutations keeping leg 1 fixed. The $T^{a}$ are color-group generators, encoding the color of each external leg $1,2,3 \ldots, n . A_{n}^{\text {tree }}$ is a tree-level color-ordered $n$-leg partial amplitude, $n_{i}$ are numerators of kinematic poles, and the sum in (51) runs over all distinct order diagrams. $p_{m}$ are particle momenta. The full tree amplitude for gluons produced because of the Unruh effect near a mini black holes can be decomposed as,

$$
\begin{aligned}
& \tilde{H}_{n}^{\text {tree }}(1,2,3, \ldots, n)=\alpha_{s}^{n-1} \Gamma_{n} \sum_{p(2,3, \ldots, n)} \operatorname{Tr}\left[T^{a_{1}} T^{a_{2}} T^{a_{3}} \ldots . . T^{a_{n}}\right] \tilde{A}_{n}^{\text {tree }}(1,2,3, \ldots, n) \\
& \tilde{A}_{n}^{\text {tree }}(1,2,3, \ldots . n)=\sum_{j} \frac{\tilde{n}_{j}}{\left(\prod_{m} \omega_{m}^{2}\right) j} \quad \Gamma_{n}=\prod_{n} N_{\omega_{n}, \text { color }}^{\text {gluon }}
\end{aligned}
$$


We conclude that the number of interactions increase near mini black holes at LHC. In fact we detect extra particles compared with our predictions based on the Unruh effect at LHC.

\section{Summary and conclusion}

We calculated the black hole final states for gluons and quarks. We also calculated the effect of information loss for gluons and quarks produced from mini black holes at LHC. Next, we determined the total cross-section for mesons produced from black holes at LHC. We derived the resulting information transformation from the collapsing matter to the state of outgoing Hawking radiation for mesons. We observed that due to the deviation of the $f$ from unity, some of information is lost in black holes. However, when we regard scalar and Dirac fields together, all of information inside the black hole can transform to outside the black hole. Then, we observed that information loss is complete in the case of excited black holes because of their unstable condition. We then considered the quantum black hole production in electron-positron annihilation modeling at $\mathrm{TeV}$ centre of mass energies. Finally we studied the gluon tree level amplitudes near mini black holes at LHC.

\section{References}

1. G.T. Horowitz and J. Maldacena. J. High Energy Phys. 2004, 008 (2004). doi:10.1088/1126-6708/2004/02/008.

2. X. Calmet, W. Gong, and S.D.H. Hsu. Phys. Lett. 668B, 20 (2008).

3. G. Dvali and M. Redi. Phys. Rev. D, Part. Fields Gravit. Cosmol. 77, 045027 (2008); B. Koch, M. Bleicher, and H. Stöcker. J. Phys. G, 34, S535 (2007). doi:10.1088/09543899/34/8/S44.;D.M. Gingrich. Available from arxiv.org/ abs/0912.0826.

4. A. Chamblin, F. Cooper, and G.C. Nayak. Phys. Rev. D Part. Fields Gravit. Cosmol. 70, 075018 (2004).

5. G.C. Nayak and J. Smith. Phys. Rev. D, Part. Fields Gravit. Cosmol. 74, 014007 (2006); H. Higgs. New Sci. 28, 12 (2006).

6. A. Chamblin, F. Cooper, and G.C. Nayak. Phys. Lett. 672B, 147 (2009).
7. Y. Ling, S. He, W. Qiu, and H. Zhang. J. Phys. A, 40, 9025 (2007). doi:10.1088/1751-8113/40/30/024.

8. D. Ahn. Phys. Rev. D Part. Fields Gravit. Cosmol. 74, 084010 (2006).

9. X.-H. Ge and Y.-G. Shen. Phys. Lett. 612B, 61 (2005).

10. D. Ahn, Y.H. Moon, R.B. Mann, and I. Fuentes-Schuller. J. High Energy Phys. JHEP0806:062 (2008); doi:10.1088/ 1126-6708/2008/06/062.; P.M. Alsing, I. Fuentes-Schuller, R.B. Mann, and T.E. Tessier. Phys. Rev. A, July 2006, arXiv:quant-ph/0603269.

11. D. Gottesman and J. Preskill. J. High Energy Phys. JHEP0403:026 (2004).

12. D. Deutsch. Phys. Rev. D, Part. Fields, 44, 3197 (1991).

13. S.W. Hawking. Phys. Rev. D, Part. Fields, 72, 084013 (2005); doi:10.1103/PhysRevD.72.084013.T. Vachaspati, D. Stojkovic, and M. Krauss. Phys. Rev. D, Part. Fields, 76, 024005 (2007); doi:10.1103/PhysRevD.76.024005.J. Zhang, Y. Hu, and Z. Zhao. Mod. Phys. Lett. A, 21, 1865 (2006). doi:10.1142/S0217732306019980 .

14. D. Ahn. J. High Energy Phys. JHEP0703:021 (2007).

15. S. Lloyd. Phys. Rev. Lett. 96, 061302 (2006). doi:10.1103/ PhysRevLett.96.061302. PMID:16605980.

16. D.E. Miller. Eur. Phys. J. C, 34, 435 (2004); doi:10.1140/ epjc/s2004-01746-0; S. Dimopoulos and G. Landsberg. Phys. Rev. Lett. 87, 161602 (2001). doi:10.1103/ PhysRevLett.87.161602. PMID:11690198.; P.C. Argyres, S. Dimopoulos, and J. March-Russell. Phys. Lett. 441B, 96 (1998).

17. S.B. Giddings and S. Thomas. Phys. Rev. D, Part. Fields, 65, 056010 (2002); A. Chamblin, F. Cooper, and G.C. Nayak. Phys. Rev. D, Part. Fields Gravit. Cosmol. 69, 065010 (2004); F. Cooper, E. Mottola, and G.C. Nayak. Phys. Lett. 555B, 181 (2003).

18. S. Dimopoulos and G. Landsberg. Phys. Rev. Lett. 87, 161602 (2001); doi:10.1103/PhysRevLett.87.161602. PMID:11690198.; P.C. Argyres, S. Dimopoulos, and J. March-Russell. Phys. Lett. 441B, 96 (1998).

19. T. Søndergaard. Nucl. Phys. B821, 417 (2009). doi:10.1016/ j.nuclphysb.2009.07.002. 